\title{
Syitem
}

des

\section{Römifhen Redhts}

non

faeintid Rernlumry,

veil. otbentlidem Wrofejïor an Der ltniveriftät Berlin.

\section{Der Pandeften}

adte, umgearbeitete $\mathfrak{A}$ uf́lage

bearbeitet

von

\section{Dr. Haul Sokolomski,}

vorm. a. o. Brofeffor an ber univerfität Bertiu

unto croentither \&rofeifor an ber univerfitat Röniguberg.

\section{3wciter Teil.}

Berlin 1912.

Berlag von Sc. W. Müller. 
Jlle Red te vorbehalten.

mon 